\title{
Carnets
}

Revue électronique d'études françaises de l'APEF

Deuxième série - 5 | 2015

Imaginaires de guerre et autres conflits

\section{Une seconde Troie}

Andromaque de Racine ou l'art de refaire la guerre

\section{Guillaume Gomot}

\section{(2) OpenEdition \\ Journals}

Édition électronique

URL : http://journals.openedition.org/carnets/358

DOI : $10.4000 /$ carnets.358

ISSN : 1646-7698

Éditeur

APEF

Référence électronique

Guillaume Gomot, "Une seconde Troie », Carnets [En ligne], Deuxième série - 5 | 2015, mis en ligne le 30 novembre 2015, consulté le 30 avril 2019. URL : http://journals.openedition.org/carnets/358 ; DOI : $10.4000 /$ carnets.358

Ce document a été généré automatiquement le 30 avril 2019.

\section{(c) (i) \&}

Carnets est mis à disposition selon les termes de la licence Creative Commons - Atribution - Pas d'utilisation commerciale 4.0 International. 


\title{
Une seconde Troie
}

\author{
Andromaque de Racine ou l'art de refaire la guerre
}

\author{
Guillaume Gomot
}

1 La liste des personnages d'Andromaque, premier chef-d'œuvre de Racine (1667), indique clairement le caractère singulier de cette pièce : les acteurs de la tragédie y sont tous, en effet, définis par leur illustre ascendance. Dès lors, l'Épire, qui tient lieu de décor à l'action, pourrait devenir « un second Ilion » (v. 564) et voir se rejouer le plus glorieux des conflits antiques qui, d'Homère à Virgile, a nourri maintes épopées fondatrices : la guerre de Troie.

2 Bellum ad infinitum: Andromaque semble attester l'existence d'un tragique de répétition, fondé sur le souvenir d'images à la fois ressassées et cristallisées, sur la pesanteur d'un passé omniprésent et sur la reprise incessante de conflits antérieurs, d'ordre amoureux ou guerrier. Mais comment les figures tragiques résistent-elles à cette position intenable, victimes d'une guerre achevée qui pourtant n'en finit pas? Si Andromaque survit à cette guerre réitérée qui précipite Oreste dans la folie, Pyrrhus et Hermione, quant à eux, y succombent, perdus respectivement dans un massacre et un suicide.

3 Il s'agira donc ici de suivre l'onde de choc provoquée par la guerre dans le poème racinien, et de dessiner les linéaments composés par cette déflagration verbale qui permet au dramaturge d'élaborer un riche imaginaire du conflit. Prêtant attention à la matière textuelle et à sa mise en forme poétique, cette étude envisagera également les horreurs de la guerre telles que Racine les met en scène avec puissance : on analysera notamment ces célèbres hypotyposes où le témoin d'un événement terrible anime son récit ou sa description avec une telle énergie qu'il en fait une image vivante du passé.

\section{Tragique de répétition}

4 Les conflits sans fin qui agitent Andromaque démontrent le principe fondateur de cette tragédie : la répétition ${ }^{1}$. La structure même de la pièce le confirme. En effet, Oreste et Pylade sont présents dans la première et la dernière scène, et la «mélancolie " initiale d'Oreste (v.17) s'accomplit dans sa folie finale, achevant pleinement l'effet d'ironie 
tragique préparé par Racine dès le deuxième vers de la pièce, où il fait dire à un Oreste enthousiaste : « Ma fortune va prendre une face nouvelle ». (v. 2)

5 Mais point de nouveauté ici : l'alpha et l'oméga du poème racinien semblent se confondre, enserrant Andromaque dans le cercle de la répétition et du souvenir incessant. «Toujours» (v. 47), «tous les jours » (v. 48), « je me souvins» (v. 49), «chaque jour » (v. 111), « plus de cent fois » (v. 115) : la scène est, d'emblée, l'espace du ressassement, des actions redondantes et des atermoiements. Le malheur s'y répand par contagion ${ }^{2}$ et c'est avec horreur que le passé s'y rejoue. Comme l'écrit Barthes (1991:52): «La durée racinienne n'est jamais maturative, elle est circulaire, elle additionne et ramène sans jamais rien transformer (...). Ce temps-répétition est naturellement celui qui définit la vendetta, la génération infinie et comme immobile des crimes. »

6 Ces vers le prouvent :

Je l'ai vu dans leurs mains quelque temps se débattre,

Tout sanglant à leurs coups vouloir se dérober,

Mais enfin à l'autel il est allé tomber. (v. 1518-1520)

La mort de Pyrrhus, décrite ici par Oreste, reproduit à l'identique le meurtre de Priam, le vieux roi de Troie tué par Pyrrhus lui-même lors du sac de la ville. C'est ainsi que L'Énéide rapporte cette mort : «(...) il traîna au pied des autels le vieillard tremblant et qui glissait dans le flot de sang de son fils, il lui saisit la chevelure de la main gauche, et, de la droite, brandissant son épée étincelante, la lui enfonça dans le flanc jusqu’à la garde. » (Virgile, $1993: 65)$

8 De Troie à l'Épire et de Virgile à Racine, le sanguinaire Pyrrhus est devenu la victime que les Grecs assassinent, mais cette inversion ne rompt pas le cercle d'une violence constante : la guerre ne connaît aucune solution de continuité et le décor d'Andromaque pourrait servir à un " second Ilion » (v. 564). Cette « seconde Troie » (v. 230) confirmerait la puissance extrême d'un cycle perpétuel de la vengeance, ce "processus infini, interminable", pour reprendre les mots de René Girard (2008: 28). La tragédie serait alors l'objet d'une sorte de contrainte ou de compulsion de répétition (la terminologie freudienne traduit ainsi Wiederholungszwang). En effet, la haine des Grecs pour Hector, malgré sa mort et l'outrage fait à son cadavre, «n'est pas encore éteinte » (v. 269) et ce feu belliqueux est avivé par la présence en Épire du fils d'Hector et Andromaque, Astyanax ${ }^{3}$.

Oui, les Grecs sur le fils persécutent le père ;

Il a par trop de sang acheté leur colère,

Ce n'est que dans le sien qu'elle peut expirer. (v. 225-227)

9 Oreste, ambassadeur des Grecs, justifie ici que « leur colère » se déporte du père vers le fils et, par une habile syllepse, il conjoint le sang métaphorique de la lignée et celui qui fut versé lors des combats. Comme le fait remarquer Jean-Pierre Landry:

(...) la parole tragique enferme chaque personnage dans la nuit de son passé : ces êtres ne sont pas seulement solidaires de leurs aïeux, ils en sont les prisonniers et les otages, condamnés à revivre leur destin. (...) ils sont esclaves de cette violence héritée, qui devient leur violence propre. (Landry, $2000: 68$ )

10 Quoi qu'il en coûte aux personnages, l'élan de la vengeance semble irrépressible pour certains, même en matière amoureuse. Comme le déclare Hermione, délaissée par Pyrrhus : «Que je me perde ou non, je songe à me venger ». (v. 1256)

11 Et le succès considérable d'Andromaque, depuis sa création en 1667, doit beaucoup, à coup sûr, à cette alliance cardinale entre la guerre et l'amour, le tragique et la galanterie, qu'a 
réussie Racine. Phœnix, le gouverneur et confident de Pyrrhus, le prouve quand il souligne la profonde immixtion des intérêts amoureux et politiques d'Hermione: "La querelle des Grecs à la sienne est liée ». (v. 1390)

Sur la scène amoureuse, les combattants sont moins nombreux que devant les murs de Troie, mais la guerre qu'ils livrent est violente et cruelle. Et, grâce à son balancement et à sa symétrie rythmique, l'alexandrin classique, dont Racine développe toute la riche prosodie, est éminemment propice à faire résonner cet affrontement des cœurs, cette guerre en sourdine où mots et regards blessent comme des armes. Des soupirs aux vociférations, la parole des personnages s'empreint souvent de la métaphore du «combat " amoureux (v. 703). Ainsi Pyrrhus est-il las de "combattre » les «cruautés " d'Andromaque (v. 292); il « croit avoir en l'amour vaincu mille ennemis » (v. 636), après avoir repoussé la veuve d'Hector et choisi d'épouser Hermione. Mais, «brûlé de plus de feux » qu'il n'en a allumés (v. 320), il devra reconnaitre que l'amour venge la défaite troyenne et qu'Andromaque est souveraine en son cœur.

Les ressources de l'imaginaire galant et son goût pour l'hyperbole trouvent donc un parfait accomplissement dans le lexique guerrier. Alliés aux mots, les regards sont aussi les acteurs d'une confrontation discrète mais féroce. "Je sais que vos regards vont rouvrir mes blessures» (v. 485), lance Oreste à Hermione, décrivant cette autre guerre, indicible, qui parcourt Andromaque et se fonde sur l'éloquence et la puissance du regard. Il s'agit, pour chacun, d'«armer [ses] yeux » (v. 459) puis d'éprouver « le pouvoir de leurs armes " (v. 534), pour se faire aimer ou pour mettre un ingrat à la torture. Dès lors, comme le montre Paul Bénichou, l'amour se mue en « un désir jaloux, avide, s'attachant à l'être aimé comme à une proie » (Bénichou, 1992 : 182).

14 L'expression du dépit et de la souffrance des personnages emploie souvent des images tout à la fois crues et cruelles (ces deux adjectifs sont issus d'un étymon commun : le sang ${ }^{4}$ ). C'est le cas de ces vers où Hermione projette de tuer Pyrrhus avant de se suicider :

Je percerai le cœur que je n'ai pu toucher, Et mes sanglantes mains, sur moi-même tournées,

Aussitôt, malgré lui, joindront nos destinées. (v. 1244-1246)

15 Ce rêve d'une union dans la mort recourt au «cœur " dans un double sens littéral et métaphorique, qu'on retrouve aussi dans ces mots d'Oreste :

L'ingrate mieux que vous saura me déchirer ;

Et je lui porte enfin mon cœur à dévorer. (v. 1643-1644)

C'est à Hermione, "l'ingrate ", que pense Oreste dans ses dernières paroles sur scène, laissant retentir ensuite l'éclat barbare de cette image où l'amour semble une guerre à mort, achevée par la possession et la destruction physiques. Le substantif polysémique cœur est ainsi étendu, outré, dans un élan quasi baroque et l'on pourrait penser, par exemple, à certains poèmes de l'Hécatombe à Diane, où d'Aubigné mêle l'analyse des sentiments à des descriptions crues, comme dans celui-ci :

J'ouvre mon estommac, une tumbe sanglante

De maux enseveliz; pour Dieu, tourne tes yeux,

Diane, et voy au fons mon cueur party en deux

Et mes poumons gravez d'une ardeur viollente, (...)

Au moins après ma fin que ton ame apaisée

Bruslant le cueur, le cors, hostie à ton courroux,

Prenne sur mon esprit un supplice plus doux,

Estant d'yre en ma vie en un coup espuisée. (Rousset, 1988 : 111) 
17 Le conflit amoureux revêt ici l'aspect d'une guerre intestine qui dévaste et détruit le corps même du poète supplicié par l'amour, à la lettre et dans tous les sens.

18 Comme une échappatoire à la crise tragique, à la guerre des regards et au vertige de la destruction, certains personnages d'Andromaque postulent la nécessité d'une rupture qui pourrait clore le cycle de la répétition. Hermione veut se «séparer » de Pyrrhus (v. 420) mais pourtant n'y parvient pas, comme le montre ce vers où Pylade la décrit « Toujours prête à partir, et demeurant toujours » (v. 131). Le chiasme et l'antithèse donnent ici corps au paradoxe d'une sorte de rupture continue.

19 Mais c'est Pyrrhus qui représente, au premier chef, l'aboutissement du désir de rompre. Car cet « enfant rebelle » de la Grèce (v. 237), « qui ne se souvient plus qu'Achille était son père » (v. 990), est prêt à épouser Andromaque et à protéger le fils d'Hector. Malgré ses revirements et le choix temporaire d'une "solution conformiste ${ }^{5}$, pour reprendre les mots de Barthes (1991: 54), Pyrrhus poursuit sa révolte, rêvant « d'oublier le passé » (v. 1344) par une amnésie salvatrice, afin d'instaurer une nouvelle Épire où les conflits et les enjeux antérieurs seront "sortis de sa mémoire" (v.1450). Il désire donc une "Légalité nouvelle (...) qui ne sera plus fondée sur le retour immuable des vengeances » (Barthes, 1991: 78-79). Mais il le paiera de sa vie. En effet, nulle sécession ne peut entièrement s'accomplir dans Andromaque, tragédie de la fidélité et du souvenir. Et c'est en fait la catastrophe de la pièce qui en constitue le seul moment de rupture, détachant brutalement les personnages des liens qui les déterminaient et les précipitant dans la mort, la folie ou la solitude.

\section{Vie des fantômes}

Le principe de réitération propre à Andromaque met en scène les acteurs des conflits passés de manière à leur donner une présence singulière : c'est comme si les fantômes de Troie et de la Grèce étaient bien vivants et, sortis du royaume d'Hadès et de Perséphone, revenaient hanter l'espace tragique.

21 Les périphrases incessantes qui définissent les personnages réveillent le souvenir des héros de la guerre: Andromaque et Astyanax sont souvent désignés par leur lien avec Hector, dont ils sont respectivement la veuve et le fils. Hermione est ainsi rapprochée d'Hélène, Oreste d'Agamemnon et Pyrrhus d'Achille. L'identité des enfants est subsumée sous le nom glorieux de leurs parents et la généalogie détermine l'équation tragique qui constitue, pour Pyrrhus, le nœud d'Andromaque :

Elle est veuve d'Hector, et je suis fils d'Achille :

Trop de haine sépare Andromaque et Pyrrhus. (v. 662-663)

Le premier alexandrin (v.662), discontinu, repose sur un parallélisme syntaxique, accentué par la virgule à la césure. Sa double construction attributive fait déjà entendre, de manière implicite, l'impossible union de la "veuve d'Hector » et du "fils d'Achille ", soumis à une discorde essentielle. Dans cette perspective, la conjonction « et " peut exprimer une relation d'opposition que le vers suivant (v. 663) développe et explique : "Trop de haine sépare Andromaque et Pyrrhus ». Mais le constat d'hostilité du premier hémistiche ("Trop de haine sépare ») est contredit par le second, qui isole et réunit dans l'espace clos de son hexamètre le couple "Andromaque et Pyrrhus ». En effet, à cet instant, Phœnix veut persuader Pyrrhus de renoncer à Andromaque et d'épouser Hermione, mais les paroles du roi d'Épire portent les signes d'une dénégation, comme 
l'atteste l'ordonnance métrique. Les propos de Pyrrhus sont démentis par la capacité paradoxale de ces vers à faire exister un couple dont la possibilité même est d'abord niée. Plus loin dans cette scène, Phœnix devra se résoudre à constater la toute-puissance de l'amour que Pyrrhus porte à Andromaque ${ }^{6}$. Mais le roi d'Épire, s'il décide d'oublier la haine guerrière qui devrait l'opposer à la veuve d'Hector, en subira finalement le choc en retour, qui le perdra, car la logique de la tragédie va l'immoler à sa mécanique infernale.

Les personnages d'Andromaque - tragédie des épigones - se comparent aux héros illustres de la guerre de Troie, pour vanter des similitudes ou désigner des dissemblances. Il est donc fréquent qu'on parle à Pyrrhus de son père Achille, et quand Oreste le rencontre (acte I, scène 2), il formule des compliments d'usage qui flattent cette ascendance célèbre, pour mieux rappeler Pyrrhus à son devoir en tant que Grec. Et Phœnix, croyant le roi d'Épire décidé à rejeter Andromaque et à épouser Hermione, proclame :

C'est Pyrrhus, c'est le fils et le rival d'Achille, (...)

Qui triomphe de Troie une seconde fois. (v. 630, 632)

La conformité à la légende lumineuse d'Achille est une vertu que Phœnix promeut. JeanPierre Vernant (1996: 43) rappelle qu'Achille est, par surcroît, "voué par avance - on pourrait dire par nature - à la belle mort ", car «il est de son vivant comme imprégné déjà par l'aura de la gloire posthume à laquelle il a toujours été promis ». Et la figure inégalable d'un père mythique dont l'éclat animerait le fils est d'autant plus vive que Phœnix, avant d'être le gouverneur de Pyrrhus, avait été celui d'Achille. Jouant avec l'onomastique, on pourrait d'ailleurs voir en Phonix l'emblème de la répétition, qui dédaigne la mort et les cendres. Mais, sans cesse comparé à un père héroïque, présent dans les paroles et les souvenirs des personnages, Pyrrhus devient son « rival » et court le risque de déchoir. Malgré la distance, le père et le fils entrent en concurrence l'un avec l'autre, comme dans cet alexandrin où l'on dit d'Oreste qu'« Il respecte en Pyrrhus Achille et Pyrrhus même » (v. 1466). La contiguïté des deux noms, de part et d'autre d'une césure effacée par l'enjambement interne, accentue la cohésion du couple père-fils : Pyrrhus ne peut échapper, malgré qu'il en ait, au poids de son sang et le nom de son père lui est irrémédiablement attaché. La "fidélité » à laquelle il est soumis lui fait éprouver, pour reprendre les mots de Barthes (1991: 50), «l'horreur même d'un engluement: il est retenu dans sa propre antériorité comme dans une masse possessive qui l'étouffe ». C'est ce que montre précisément le vers de Racine : « (...) en Pyrrhus Achille (...) ».

Plus qu'Achille encore, l'ombre d'un guerrier défunt recouvre la tragédie de la première à la dernière scène : c'est Hector, à qui la pièce de Racine redonne vie ${ }^{7}$. Déplorant la ruine de Troie, Andromaque chante sa douleur :

Non, vous n'espérez plus de nous revoir encor,

Sacrés murs que n'a pu conserver mon Hector ! (v. 335-336)

Pleurant son époux, elle ranime sa gloire et contredit le sens apparent de ces deux vers fondés sur des tournures négatives. La rime dévoile alors le dessein véritable du poème racinien qui, refusant sa disparition, rappelle sur scène le prince troyen: "encor / Hector ». Les stratégies rhétoriques et dramaturgiques qui donnent corps à ce fantôme sont multiples. Ainsi, lorsqu'Andromaque fait comprendre qu'elle pourrait choisir de mourir, elle prétend « revoir » (v. 378) et « rejoindre [son] époux» (v. 924), confirmant par ces verbes concrets sa conviction d'une présence réelle d'Hector. Cette dernière est renforcée par les passages où Andromaque s'adresse directement à son époux défunt (v. 940-946) puis rapporte ses paroles, faisant entendre sur scène la voix du guerrier (v. 1021-1026). A la fin de l'acte III, balançant et ne sachant quelle décision prendre 
- « périr ou régner » (v. 968), selon les mots de Pyrrhus - c'est vers Hector qu'Andromaque se tourne, achevant l'acte par cet alexandrin : "Allons sur son tombeau consulter mon époux ». (v. 1048)

Après une mystérieuse délibération auprès du cénotaphe d'Hector, ce tombeau vide érigé à la mémoire de l'absent-présent, Andromaque réapparait, au début du quatrième acte, sûre du choix que son époux lui « a commandé lui-même » (v. 1098). Jean Rohou (1991: 61) souligne ainsi la puissance de cette figure à la fois tutélaire et dominatrice : «Oreste, Hermione, Pyrrhus croient agir sans voir que, de fil en aiguille, ils sont tous conduits par l'attrait irrésistible qu'à travers Andromaque exerce sur la chaîne entière Hector, le mort, lieu de la valeur inaccessible en ce monde.»

Dans la guerre continue d'Andromaque, Hector joue donc un rôle actif, vivant à travers sa veuve $^{8}$, représentant "le souvenir de Troie» (v. 1438) et de ses morts. Mais son fils Astyanax, qu'on ne voit jamais sur scène, est lui aussi consubstantiel à cet art de refaire la guerre dont Andromaque serait le traité poétique. Son nom signifie d'ailleurs « maître de la cité » : par lui Troie peut continuer. Voici comment Oreste le définit :

(...) l'ennemi de la Grèce,

Astyanax, d'Hector jeune et malheureux fils. (v. 70-71)

On remarque que « d'Hector », le complément du nom «fils », est délibérément antéposé, pour rejoindre «Astyanax » dans le premier hémistiche et créer de cette manière un effet d'assimilation. La proximité phrastique d'«Hector» et de "jeune", que sépare avec ténuité une césure enjambée, renforce en outre la comparaison. Jean Starobinski l'énonce avec clarté : «En Astyanax, Andromaque revoit Hector ; tout le passé redevient présent » (2008 : 79). Car Astyanax est « l'image d'Hector » (v. 1016), et dans le regard de sa mère, la confusion est parfois entière, comme le souligne Pyrrhus qui rapporte ici les paroles d'Andromaque :

C'est Hector, disait-elle, en l'embrassant toujours ;

Voilà ses yeux, sa bouche, et déjà son audace ;

C'est lui-même ; c'est toi, cher époux, que j'embrasse. (v. 652-654)

Cette similitude fantasmatique, qui superpose deux images et nie toute loi historique, semble soustraire Astyanax aux contraintes temporelles et autorise Andromaque à déclarer, sans crainte des connotations incestueuses, qu'il lui « aurait tenu lieu d'un père et d'un époux» (v. 279). Astyanax, en qui survivent «tous ses aïeux» (v. 1028) et qui pourrait à l'avenir venger Troie, constitue le point de fuite de la tragédie. Cette figure du point de fuite peut être lue de diverses manières. En effet, si l'on réunit les dimensions spatiales et temporelles, Astyanax, vivant mais exclu de la scène présente, est bien le point d'intersection des lignes de fuite de la tragédie: il est l'enjeu des tensions dramatiques, casus belli à lui seul, mais il concentre aussi l'idée même d'un avenir, d'un temps neuf, affranchi des lois pesantes de la répétition et de la guerre. Étymologiquement, l'enfant Astyanax - in-fans sans parole - échappe à la fatalité tragique, au fatum - ce qui a été prédit ${ }^{9}$. Il porte en lui l'espoir d'une nouvelle royauté et Racine s'est certainement rappelé que le fils d'Hector, d'après la Franciade de Ronsard, aurait été à l'origine de la monarchie française.

31 Si Hector tient lieu de point de vue dans Andromaque, en tant que regard extérieur qui préexiste à la tragédie et la détermine, Astyanax en est aussi le point de fuite pour une autre raison. En effet, Daniel Arasse (1999) a brillamment montré que, dans les tableaux de la Renaissance italienne représentant l'Annonciation, la science de la perspective ouvre l'espace à la transcendance divine et à l'avènement futur du Christ, qu'elle 
préfigure souvent dans l'image, notamment par le point de fuite ; et c'est donc grâce aux ressources de l'esthétique que l'on pourrait comprendre, dans sa plus large extension, cette notion du point de fuite littéraire. Ce n'est d'ailleurs pas un hasard si Andromaque a parfois été considérée comme une figure mariale, Astyanax étant lui-même comparé au Sauveur.

Si des personnages absents - Achille, Hector, Astyanax - occupent pourtant la scène, on peut noter également que certains acteurs de la tragédie la quittent en fantômes : c'est le cas de Pyrrhus et d'Hermione, dont les images hantent l'esprit perdu d'Oreste dans la dernière scène. Cette traversée des mondes est réciproque et elle éclaire une dernière fois la vie des fantômes raciniens.

\section{Poétique des ruines}

L'imaginaire de la guerre, dans Andromaque, donne une forme littéraire aux ruines. Et l'évocation d'une poétique des ruines renvoie, bien sûr, aux réflexions de Diderot face aux œuvres d'Hubert Robert, notamment dans le Salon de 1767 - un siècle exactement après la création d'Andromaque. Pour Diderot, « la peinture des ruines (...) a sa poétique » (2000 : 700) et elle suscite maintes considérations philosophiques :

Les idées que les ruines réveillent en moi sont grandes. Tout s'anéantit, tout périt, tout passe, il n'y a que le monde qui reste, il n'y a que le temps qui dure. Qu'il est vieux, ce monde! Je marche entre deux éternités. De quelque part que je jette les yeux, les objets qui m'entourent m'annoncent une fin et me résignent à celle qui m'attend. (Diderot, 2000 : 701)

Mais comment Andromaque développe-t-elle sa poétique des ruines? Décombres de Troie, débâcle amoureuse, chute des êtres : Racine invente une tragédie des vestiges et des restes . Pour Barthes (1991 : 45-46), c'est le concept de revirement qui s'impose, défini de la sorte : " il met les choses de haut en bas, la chute est son image (il y a probablement chez Racine une imagination descensionnelle) ». Une rapide enquête lexicale démontrerait la fréquence et la force de ce substantif - reste - dans cette pièce de l'après-guerre. En effet, Astyanax est décrit comme le "Reste de tant de rois sous Troie ensevelis» (v. 72). En position initiale, «Reste» informe l'alexandrin, en provoquant la répétition du groupe consonantique [rst] dans « rois sous Troie » et en accompagnant les échos sémantiques (avec « ensevelis ») et sonores (entre « rois » et « Troie »). Andromaque présente ailleurs son fils comme « Le seul bien qui [lui] reste et d'Hector et de Troie » (v. 262). Accentué à la césure et employé ici sous sa forme verbale, c'est le même mot qui figure l'idée des vestiges, à de nombreuses reprises dans la tragédie, avec des analogies de construction parfois frappantes (« (...) Sauve tout ce qui reste et de Troie et d'Hector», v. 599) ou des répétitions insistantes, comme dans ces trois alexandrins décrivant Astyanax :

Il est du sang d'Hector, mais il en est le reste ;

Et pour ce reste enfin j'ai moi-même, en un jour,

Sacrifié mon sang, ma haine, et mon amour. (v. 1122-1124)

On peut aussi remarquer que le discours amoureux lui-même fait usage du terme, dans des expressions comme "un reste de tendresse» (v. 477 et 702) ou «un reste d'espérance » (v. 498).

Disséminés dans le poème racinien, les restes de l'amour et de la guerre avivent le souvenir de la destruction de Troie, un an auparavant, qui traverse plusieurs scènes. Ainsi, comme l'écrit Marcel Gutwirth (1970: 48), «Troie flambe une dernière fois, dans 
Andromaque, dans le cœur de ceux qui n'en recueillent la succession sanglante que pour en revivre le bilan désastreux ». Pyrrhus, le premier, rappelle ces instants où « La victoire et la nuit (...) excitaient au meurtre » les Grecs (v. 211-212) et développe l'imaginaire des ruines de Troie :

Je ne vois que des tours que la cendre a couvertes,

Un fleuve teint de sang, des campagnes désertes,

Un enfant dans les fers; et je ne puis songer

Que Troie en cet état aspire à se venger. (v. 201-204)

Les visions de la ville détruite, abandonnée, et des conséquences de la guerre précèdent, dans la pièce, celles de la chute de Troie et des massacres qu'elle causa. Remontant ensuite jusqu'à la source vive et sanglante de la tragédie, Andromaque, fidèle à la mémoire de Troie et meurtrie par l'idée d'épouser un Grec, fait revivre ces heures terribles. Dès lors, c'est, pour reprendre l'image de Jean Rousset (1953 : 247), le «bassin des années profondes qui se débonde» et répand sur scène l'horreur "d'un passé monstrueusement amassé et tendu à éclater » :

J'ai vu mon père mort et nos murs embrasés ;

J'ai vu trancher les jours de ma famille entière,

Et mon époux sanglant traîné sur la poussière. (v. 928-930)

C'est la force du regard d'Andromaque - ceil vivant créé par la puissance poétique de Racine - qui dit la guerre. Le chant de douleur de la veuve d'Hector ${ }^{10}$ alterne des moments de déploration élégiaque ${ }^{11}$ et de grande violence. Le poème devient furieux quand le souvenir des dévastations éclate sans retenue :

Dois-je oublier Hector privé de funérailles,

Et traîné sans honneur autour de nos murailles?

Dois-je oublier son père à mes pieds renversé,

Ensanglantant l'autel qu'il tenait embrassé ?

Songe, songe, Céphise, à cette nuit cruelle

Qui fut pour tout un peuple une nuit éternelle ;

Figure-toi Pyrrhus, les yeux étincelants,

Entrant à la lueur de nos palais brûlants,

Sur tous mes frères morts se faisant un passage,

Et de sang tout couvert échauffant le carnage ;

Songe aux cris des vainqueurs, songe aux cris des mourants,

Dans la flamme étouffés, sous le fer expirants ;

Peins-toi dans ces horreurs Andromaque éperdue :

Voilà comme Pyrrhus vint s'offrir à ma vue. (v. 993-1006)

Si Andromaque semble alors s'abandonner à son souvenir, saisie par un vertige mémoriel, il n'est pas moins vrai que, d'emblée, son trouble s'exprime ici avec une grande clarté. Comme le note Leo Spitzer (1993: 279), c'est « un égarement qui n'oublie pas d'ajuster artistement les plis de l'éloquence», avec une musicalité réglée et une ordonnance proprement classique.

Cette célèbre hypotypose est parcourue par l'onde de choc de la "nuit éternelle " qu'Andromaque se remémore. «Des visages qui apparaissent dans la nuit, à la lumière des flammes : il semble que ce soit là un thème favori de la rêverie racinienne ", écrit Jean Starobinski (2008: 81), dont l'observation trouve ici un exemple accompli et terrible. A l'horreur du massacre correspond l'éclat verbal et prosodique qui anime ces vers. Les répétitions et les modulations lexicales renforcent un effet de saturation exceptionnel, qui n'est pas pour rien dans la gloire unique de cet extrait : au cœur d'une nuit déchirée par les flammes et teintée de sang résonnent les cris réunis « des vainqueurs » et « des 
mourants", dans un vers dont l'ampleur volumétrique semble d'ailleurs imiter la prodigieuse intensité du son. L'acuité visuelle et sonore d'Andromaque est telle qu'elle en devient douloureuse. Et cet excès sensible, qui ravive la ruine de Troie, donne à ce tableau l'aspect d'une épouvantable fantasmagorie, dans tous les sens du terme. En effet, scandé par des impératifs («Songe, Figure-toi, Peins-toi »), ce passage projette avec éclat, sur la surface textuelle et sur ses "grands pans de nuit» (Foucault, 1988:263), des images effrayantes qui blessent Andromaque à nouveau, tandis qu'elle les profère. La richesse harmonique de ces vers et la vigueur de leur cadence accentuent ainsi l'efficace du discours d'Andromaque, autrement dit "les plis de l'éloquence ». La reprise régulière et l'entrelacement de groupes consonantiques ${ }^{12}$ constitués d'une occlusive et d'un « $\mathrm{r}$ » forment, en outre, une sorte de basse continue qui dit l'horreur de la «nuit cruelle », dont le grondement, comme une sourde vibration, est encore perceptible.

Et c'est tout l'art de l'actio oratoire qui permettra ensuite aux vers de Racine de s'animer sur scène, déployant alors leur forme essentiellement dramatique. Mais on constate aisément que le poème racinien possède tous les caractères d'une "écriture à haute voix », pour employer une formule de Barthes (2000: 128). Il n'attend plus qu'une actrice capable de l'incarner, en suivant tous ses "incidents pulsionnels ", en prêtant son corps et sa voix à ce « langage tapissé de peau » où l'on peut « entendre le grain du gosier, la patine des consonnes, la volupté des voyelles, toute une stéréophonie de la chair profonde » (ibid. : 128).

42 Mais le chant des ruines obéit parfois, dans la pièce, à une visée rhétorique différente. Quand Hermione décrit les horreurs de la guerre, le lamento cristallin et les airs pathétiques d'Andromaque font place à une violente dénonciation ironique :

Du vieux père d'Hector la valeur abattue

Aux pieds de sa famille expirante à sa vue,

Tandis que dans son sein votre bras enfoncé

Cherche un reste de sang que l'âge avait glacé ;

Dans des ruisseaux de sang Troie ardente plongée ;

De votre propre main Polyxène égorgée

Aux yeux de tous les Grecs indignés contre vous :

Que peut-on refuser à ces généreux coups ! (v. 1333-1340)

Désirant outrager le "perfide» (v.1375) Pyrrhus, qui ne l'épousera pas, Hermione développe ici, et dans toute la scène, un discours fondé sur de nombreuses antiphrases. Elle renverse les valeurs héroïques relatives à la guerre : le prestige des hauts faits (les gesta) n'est que lâcheté sanguinaire; et les " généreux coups » de Pyrrhus ne démontrent qu'une cruauté aveugle. La colère d'Hermione, qui démythifie la guerre de Troie, suscite ainsi une ironie venimeuse dont on trouvera de semblables exemples chez Voltaire.

Le souvenir du sac de Troie, qui diapre d'un terrible éclat les vers d'Andromaque et d'Hermione, pourrait, par ailleurs, nous faire songer à ces «énigmatiques tendances masochistes du moi » dont Freud dessine les contours dans Au-delà du principe de plaisir (2001b : 57). Fondant notamment ses analyses sur les névroses traumatiques des vétérans de la Grande Guerre, Freud constate, en effet, que « la compulsion de répétition ramène aussi des expériences du passé qui ne comportent aucune possibilité de plaisir » (ibid.: 66), comme semble le démontrer Andromaque, rappelant son malheur, la mort d'Hector et la ruine de Troie, à la manière des cauchemars qui hantent les rescapés. Empreintes de fascination et de dégoût, des réminiscences guerrières semblables sont également étudiées par Freud dans ses Considérations actuelles sur la guerre et sur la mort, où il remarque, en 1915, que le conflit mondial « nous dépouille des couches récentes déposées 
par la civilisation et fait réapparaitre en nous l'homme des origines » (2001a: 46), dont l'inconscient "est plein de désirs meurtriers sanguinaires » (ibid. : 45). Les combattants déchaînés deviennent "une bande d'assassins " (ibid. : 43) et, comme Pyrrhus à Troie, courent le risque de l'inhumain: "Cette guerre a suscité notre désillusion pour deux raisons : la faible moralité, dans leurs relations extérieures, des États qui se comportaient à l'intérieur comme les gardiens des normes morales et, chez les individus, une brutalité de comportement, dont on n'aurait pas cru que, participant de la plus haute civilisation humaine, ils fussent capables. » (ibid. : 18)

On pourrait aussi, dans cette perspective, penser à la pièce que Hofmannsthal publie à l'issue de la guerre, L'Homme difficile (1919), où la dramaturgie est minée par le retour insidieux du conflit. "Draußen»: c'est ainsi que Hofmannsthal désigne le champ de bataille et les tranchées ${ }^{13}$. Le souvenir est donc spatialisé, tel un décor, un double fond scénographique dévoilant par instants son horreur. Sur cette scène difficile, le langage même est attaqué : l'interlocution se délite, les signes s'opacifient et les mots se font flatus vocis, au contraire de la langue racinienne, qui conserve splendeur et maîtrise même pour dire l'innommable.

Et si Racine prouve que l'épopée troyenne fut un carnage, il laisse toutefois sourdre à plusieurs reprises dans la pièce l'idée d'une relève de Troie. Oreste déclare qu'on voit Pyrrhus « du sang troyen relever le malheur » (v. 152), car le jeune Astyanax, sauvé de l'écroulement et du massacre, pourrait « voir avec lui renaître tant de rois » (v. 1071) s'il régnait un jour. Le fils d'Achille le confirme à Andromaque :

Votre Ilion encor peut sortir de sa cendre;

Je puis, en moins de temps que les Grecs ne l'ont pris,

Dans ses murs relevés couronner votre fils. (v. 330-332)

L'emploi réitéré de ce verbe, que l'on trouve aussi dans « je relevais Troie » (v. 611) et « Il veut que Troie encor se puisse relever» (v.1051), montre son importance dans la tragédie. Relever Troie, la faire " sortir de sa cendre » et conjurer son effondrement, c'est procéder, mutatis mutandis, à une sorte d'Aufhebung ${ }^{14}$ des ruines de la guerre. Ce célèbre concept hégélien, appliqué à l'univers tragique de Racine, fait entendre la polysémie d'un substantif qui a d'ailleurs pu être rendu en français par l'idée de relève, notamment sous la plume de Jean-Luc Nancy ${ }^{15}$.

Si Astyanax, point de fuite de la tragédie, est l'espoir d'une nouvelle Troie, il n'est toutefois pas moins vrai que la figure de la chute détermine Andromaque. La ruine des êtres est révélée par le sentiment de déchéance propre à plusieurs acteurs du drame : Oreste ne peut rivaliser avec Agamemnon, ni même Pyrrhus avec Achille; quant à Hermione, elle déplore amèrement la faiblesse de son empire sur les autres personnages, alors que sa mère Hélène "en sa faveur arma la Grèce entière " (v. 1478) et provoqua "dix ans de combats " (v. 1479). Elle doute même de sa propre volonté et, dans un passage relevant d'un jeu dialectique entre la contrainte et la liberté personnelle, elle se demande si elle désire vraiment le meurtre de Pyrrhus : «(...) le vouloir? Hé quoi ? c'est donc moi qui l'ordonne? » (v. 1421)

Le sujet tragique - « moi » - est lui aussi soumis à la poétique des ruines. Il court le risque de l'écroulement et de la décomposition. Et Racine développe alors, pour reprendre les mots de Jean Rohou (1991: 63), ce «tragique de déchéance avide et impuissante analysé par Pascal dès 1660 et qui exprime la vision qu'ont de l'homme la grande majorité des écrivains à partir de 1666 \%. Si Oreste, dès la première scène, est présenté comme une «âme ensevelie » (v. 18) sous le poids de la «mélancolie » classique (v.17), c'est qu'il 
constitue l'exemple absolu de la perte de soi et des ravages de la fureur qui met en pièces l'identité. Son ami Pylade le lui fait remarquer :

Modérez donc, Seigneur, cette fureur extrême.

Je ne vous connais plus; vous n'êtes plus vous-même. (v. 709-710)

Comme l'écrit Michel Foucault (1988: 267), Oreste démontre, « dans le Délire », la vérité d'une " passion qui a trouvé avec la folie la perfection de son achèvement ». Dans la scène finale, tandis qu'il «perd le sentiment» (v. 1645) et que sa conscience se trouble (« (...) suis-je Oreste enfin? », v. 1568), des visions l'assaillent, où le sang du meurtre de Pyrrhus et du suicide d'Hermione semble s'agréger à celui des massacres de la guerre :

Dans leur sang, dans le mien, il faut que je me noie. (...)

Dieux ! quels ruisseaux de sang coulent autour de moi ! (v. 1622, 1628)

51 Perdu dans la nuit de la folie et pourchassé par les terrible Érinyes, ces divinités vengeresses aux cheveux entrelacés de serpents, Oreste clôt la pièce par l'image d'une scission définitive, d'un délabrement majeur, celui des décombres de son être.

Au terme de cette étude, il convient d'insister sur la puissance poétique d'Andromaque qui, en 1667, constitue le premier sommet du théâtre racinien. L'art de dire la guerre et de la refaire continuellement est soutenu par le tissage serré, dans l'étoffe du texte, des mêmes motifs et des mêmes matières : les larmes, le sang, le feu et la cendre.

Plutôt que d'appauvrir la pièce, la simplicité lexicale d'Andromaque en fait, au contraire, une œuvre incantatoire et pure, et le premier exemple achevé, chez Racine, de poésie élémentaire. Pour Jean Rohou (2000: 79-80), cette « sobriété » entraîne des répétitions qui favorisent « (...) l'ambivalente polysémie de la langue classique : un mot utilisé au sens figuré reste encore imprégné de son emploi au sens propre peu auparavant, surtout quand cette contamination est renforcée par la constante comparaison entre les deux domaines ».

Parmi d'autres, la quatrième scène du premier acte montre ainsi l'imbrication de ces figures poétiques essentielles et les riches relations de sens qu'elles créent: le schème liquide qui définit Andromaque (" pleurer mon époux ", " vos larmes », " en repassant les eaux », « les pleurs qu'ils ont versés ») contraste avec la tonalité guerrière du discours de Pyrrhus $^{16}$, où domine la substance ignée («Leur haine (...) n'est pas encore éteinte», " voir mon palais en cendre ", " de regrets consumé », «Brûlé de plus de feux que je n'en allumai », «tant d'ardeurs inquiètes »). L'imaginaire racinien semble alors conjoindre les larmes et le feu dans le sang qui parcourt la scène. Le sang, où coexistent la liquidité des larmes et l'ardeur rougeoyante du feu, signe obsédant des combats et de la chute de Troie, circonscrit la tragédie de sa marque vermeille : il envahit l'âme d'Oreste en coulant par ruisseaux lors de son hallucination finale (acte $V$, scène 5), mais hantait déjà Pyrrhus au début de la pièce, à travers la double image du «fleuve teint de sang » (v. 202) - souvenir de Troie - et de l'assassinat éventuel d'Astyanax («Que (...) Dans le sang d'un enfant je me baigne à loisir?", v. 215-216). Et la force du poème est amplifiée par les liens féconds noués par Racine entre le sens littéral et le sens métaphorique de ces vocables premiers, confondus parfois dans de brillantes syllepses. Les vers qui suivent relient ces images élémentaires ; Andromaque y renvoie Pyrrhus vers Hermione :

Troie, Hector, contre vous, révoltent-ils son âme?

Aux cendres d'un époux doit-elle enfin sa flamme?

Et quel époux encore ! Ah! souvenir cruel!

Sa mort seule a rendu votre père immortel ;

Il doit au sang d'Hector tout l'éclat de ses armes,

Et vous n'êtes tous deux connus que par mes larmes. (v. 357-362) 
Cendres-sang, flamme-éclat-larmes: les paronomases tendent à unifier ces figures tragiques qui donnent à Andromaque sa lumineuse cohésion et en font un chant de ruines.

En définitive, pour Racine, l'art de refaire la guerre consiste en une reprise et une recréation des souvenirs de Troie. Poussées à leur point d'incandescence et soutenues par une langue maîtrisée mais incendiaire, ces images produisent le premier grand «flamboiement tragique » du théâtre racinien (Starobinski, 2008: 82), qui, dès lors, ne cessera plus de se développer, jusqu'à Athalie. La puissance d'embrasement des tragédies de Racine n'a donc pas fini, pour reprendre en la détournant la critique de Bossuet, de mettre « en feu tout le parterre et toutes les loges » (Bossuet, 1818: 542)!

\section{BIBLIOGRAPHIE}

ARASSE, Daniel (1999). L'Annonciation italienne. Une histoire de perspective. Paris : Hazan.

BARTHES, Roland (1991). Sur Racine [1963]. Paris : Seuil.

BARTHES, Roland (2000). Le Plaisir du texte [1973] précédé de Variations sur l'écriture. Paris : Seuil.

BENICHOU, Paul (1992), Morales du grand siècle [1948]. Paris : Gallimard.

BOSSUET, Jacques-Bénigne (1818). Maximes et réflexions sur la comédie [1694], in Cuvres,

tome XXXVII. Versailles : J. A. Lebel, pp. 535-621.

DECLERCQ, Gilles (2010). « Astyanax : Qui moriturus... », in Georges Banu (éds.) L'Enfant qui meurt. Motif avec variations. Lavérune : Éditions L’entretemps, pp. 81-97.

DIDEROT, Denis (2000). Salon de 1767, in Euvres, tome IV, Édition de L. Versini. Paris : Robert

Laffont.

FORESTIER, Georges (2006). Jean Racine. Paris : Gallimard.

FOUCAUlT, Michel (1988). Histoire de la folie à l'âge classique [1972]. Paris : Gallimard.

FREUD, Sigmund (2001a). Considérations actuelles sur la guerre et sur la mort [1915], in Essais de psychanalyse. Paris : Payot, pp. 9-46.

FREUD, Sigmund (2001b). Au-delà du principe de plaisir [1920], in Essais de psychanalyse. Paris : Payot, pp. 47-128.

GIRARD, René (2008). La Violence et le sacré [1972]. Paris : Hachette Littératures.

GUTWIRTH, Marcel (1970). Jean Racine. Un itinéraire poétique. Presses de l'Université de Montréal.

HEGEL, Georg Wilhelm Friedrich (1972). Science de la logique [1812]. Paris : Aubier-Montaigne.

HOFMANNSTHAL, Hugo von (2007). L'Homme difficile [1919]. Lagrasse : Verdier.

LANDRY, Jean-Pierre (2000). « Andromaque ou le devoir de mémoire », in Jean-Pierre Landry et

Olivier Leplatre (éds.). Présence de Racine. Lyon : C.E.D.I.C., n 16, pp. 67-78.

NANCY, Jean-Luc. (1973). La Remarque spéculative (un bon mot de Hegel). Auvers-sur-Oise : Galilée. 
RACINE, Jean (1999). CEuvres complètes, tome I, Édition de Georges Forestier. Paris : Gallimard, «Bibliothèque de la Pléiade».

ROHOU, Jean (1991). L'Évolution du tragique racinien. Paris : SEDES.

ROHоU, Jean (2000). Jean Racine : Andromaque. Paris : PUF.

ROUSSET, Jean (1953). La Littérature de l'âge baroque en France - Circé et le paon. Paris : José Corti.

ROUSSET, Jean. (1988), Anthologie de la poésie baroque française [1961], tome 2. Paris : José Corti.

SPITZER, Leo (1993). « L'effet de sourdine dans le style classique : Racine » [1931], in Études de style. Paris : Gallimard, pp. 208-335.

STAROBINSKI, Jean (2008). « Racine et la poétique du regard » [1957], in L’Eil vivant. Paris :

Gallimard, pp. 71-92.

TOMICHE, Anne (2008). « Histoire de répétition », in Jean-Paul Engélibert et Yen-Mai Tran-Gervat (éds.). La Littérature dépliée : reprise, répétition, réécriture. Presses Universitaires de Rennes, pp. 19-31.

VERNANT, Jean-Pierre (1996). « La belle mort et le cadavre outragé » [1982], in L'Individu, la mort, l'amour. Soi-même et l'autre en Grèce ancienne. Paris : Gallimard, pp. 41-79.

VIRGILE (1993), L'Énéide. Paris : Garnier.

\section{NOTES}

1. Pour une histoire synthétique de la répétition littéraire, voir notamment Anne Tomiche (2008).

2. «Tu m'apportais, cruel, le malheur qui te suit », dit Hermione à Oreste (v. 1556).

3. Concernant le personnage d'Astyanax, voir notamment Gilles Declercq (2010).

4. En effet, crudus et crudelis sont reliés à cruor, le sang en latin.

5. J'ai songé, comme vous, qu'à la Grèce, à mon père, / À moi-même, en un mot, je devenais contraire. (v. 609-610)

6. Vous aimez : c'est assez. (v. 685)

7. La première mention apparaît au vers 71 et la dernière au vers 1592.

8. Quoi donc ? as-tu pensé qu'Andromaque infidèle / Pût trahir un époux qui croit revivre en elle

(...) ? (v. 1077-1078)

9. Ces deux mots, enfant et fatalité, proviennent du latin fari, qui signifie parler, dire.

10. ô cendres d'un époux ! ô Troyens ! ô mon père ! / Ô mon fils, que tes jours coûtent cher à ta mère! (v. 1045-1046)

11. Concernant le ton élégiaque d'Andromaque, proche de celui des Héroïdes d'Ovide, voir Georges Forestier (2000 : 303-306).

12. Par exemple : « privé, traîné, figure, brûlants, cris ».

13. Jean-Yves Masson a choisi de traduire ce mot, qui signifie dehors, par là-bas.

14. Sur les sens contraires d'aufheben, qui peut signifier à la fois conserver et supprimer, voir Hegel (1972).

15. Voir Jean-Luc Nancy (1973).

16. Son nom même est lié au feu puisqu'il signifie « le roux ». 


\section{RÉSUMÉS}

Définis par leur ascendance illustre, les personnages d'Andromaque, premier chef-d'œuvre de Racine (1667), évoquent souvent la possibilité d'un « second Ilion ». Le dramaturge semble ainsi élaborer une tragédie de la répétition, fondée sur le souvenir d'images à la fois ressassées et cristallisées, sur la pesanteur d'un passé omniprésent et sur la reprise incessante de conflits antérieurs, d'ordre amoureux ou guerrier. Mais comment les figures tragiques résistent-elles à cette position intenable? Il s'agira ici de suivre l'onde de choc provoquée par la guerre dans le poème racinien et de dessiner les linéaments composés par cette déflagration verbale qui permet à Racine d'animer avec puissance l'imaginaire du conflit.

Defined by their well-known ancestors, the characters in Andromache (1667) - Racine's first masterpiece - often evoke the possibility of a "second Ilium". In this play, the author develops the theme of repetition, thanks to memories of both hackneyed and crystallized images, an omnipresent and burdensome past and overused past conflicts about love and war. How can tragic figures resist this untenable position? It will be necessary to focus on war and the shock waves it provoked before sketching the outline of this verbal explosion that enabled Racine to voice out his powerful vision of war.

\section{INDEX}

Keywords : Racine (Jean), tragedy, Andromache, Trojan War, repetition

Mots-clés : Racine (Jean), tragédie, Andromaque, guerre de Troie, répétition

\section{AUTEUR}

\section{GUILLAUME GOMOT}

Centre de Recherche sur les Images et leurs Relations (Paris III)

Guillaume.Gomot@ac-strasbourg.fr 